\title{
Estimating an empirical equation connecting subgrade reaction modulus with cone penetration test for sandy soils
}

\author{
Abdelaziz Ahmed Bohagr 1, Ghassan Ahmed El gehani 1, Mohammed Mahmoud Boudejaja 1, * and Mustafa M \\ Amami 2
}

${ }^{1}$ Civil Engineering Department, University of Benghazi, Libya.

${ }^{2}$ Engineering Surveying \& Space Geodes, University of Benghazi, Libya.

Global Journal of Engineering and Technology Advances, 2021, 09(02), 001-008

Publication history: Received on 05 September 2021; revised on 28 October 2021; accepted on 30 October 2021

Article DOI: https://doi.org/10.30574/gjeta.2021.9.2.0127

\begin{abstract}
In geotechnical engineering, the coefficient of subgrade reaction is regarded as one of the most important parameters used for describing the interaction of soil and structure as well as describing some soil characteristics, subgrade reaction coefficient can be calculated theoretically using many different formulas, laboratory via specific well-known tests, and in site through field plate loading test. On the other hand, the cone penetration test is one of the most frequently used field tests to investigate the soil. The lately carried out researches showed a good relation between the subgrade coefficient and the tip resistance collected from the CPT, but the results obtained from the proposed method are still doubtable.

In this paper, fifteen plate load tests and thirty CPTs, already collected for private site investigation project, have been used for finding the best fit equation connecting the subgrade reaction coefficient Ks with the tip resistance qc. The finds of the established equation have been compared extensively with those of other well-known related equations. The results show the ability of the concluded equation to get Ks results in the acceptable range of sandy soils. However, the depth and shape effect on the suggested formula need further investigations since all the plate load tests in this project have been carried out on the soil surface with a $45 \mathrm{~cm}$ diameter circular plate.
\end{abstract}

Keywords: Cone penetration test CPT; Module of subgrade reaction Ks; Plate Load Test; Tip resistance qc

\section{Introduction}

Module of subgrade reaction $\mathrm{K}_{\mathrm{s}}$ is one of the most important coefficients used in the structural analysis of the foundations, this coefficient concerns the structural engineering as well as the geotechnical engineering, the main concept of this coefficient is that the soil under the loaded area acts like elastic springs to resist the vertical deformation exerted by the load on the soil. This concept transformed to a fundamental module established by Winkler [1] which state that:

$$
\mathrm{K}_{\mathrm{s}}=\frac{\sigma}{\delta}
$$

Where;

$\mathrm{K}_{\mathrm{s}}$ is the subgrade coefficient $\left(\mathrm{kN} / \mathrm{m}^{3}\right)$

\footnotetext{
${ }^{*}$ Corresponding author: Mohammed Mahmoud Boudejaja

Civil Engineering Department, Faculty of Engineering, University of Benghazi, Libya.

Copyright $(2021$ Author(s) retain the copyright of this article. This article is published under the terms of the Creative Commons Attribution Liscense 4.0.
} 
$\sigma$ is the stress exerted on the soil $\left(\mathrm{kN} / \mathrm{m}^{2}\right)$

$\delta$ is the vertical deflection due stress (m)

In the light of Winkler's theory, a series of studies on this coefficient were conducted by Biot [2]. Terzaghi [3]. Vesic [4]. Meyerhof and Baike [5]. Selvadurai [6]. and Bowles [7, 8]. And several equations were provided depending on some different variables such as the modulus of elasticity of the foundation, the shape, the depth of foundation, and the elastic characteristics of the soil such as the modulus of elasticity and Poisson ratio, these equations are given in Table (1)

Beside these equations the coefficient of subgrade reaction can be determined in situ by applying plate load test, or it can be provided experimentally by consolidation test, triaxial test, and CBR.

Table 1 Some different formulas to calculate the modulus of subgrade reaction, ks.

\begin{tabular}{|c|c|c|c|}
\hline No. & Investigator & Year & Suggested formula \\
\hline 1 & Winkler & 1867 & $k_{s}=\frac{q}{\delta}$ \\
\hline 2 & Biot & 1937 & $k_{s}=\frac{0.95 E_{s}}{B\left(1-\mu^{2}\right)}\left[\frac{B^{4} E_{s}}{\left(1-\mu^{2}\right) E_{I}}\right]^{0.108}$ \\
\hline 3 & Terzaghi & 1955 & $k_{s f}=k_{s p}\left(\frac{B+B_{1}}{2 B}\right)^{2}$ \\
\hline 4 & Vesic & 1961 & $k_{s}=\frac{0.65 E_{s}}{B\left(1-\mu^{2}\right)} \sqrt[12]{\frac{B^{4} E_{s}}{E_{I}}}$ \\
\hline 5 & Meyerhof and Baike & 1965 & $k_{s}=\frac{E_{s}}{B\left(1-\mu^{2}\right)}$ \\
\hline 6 & Selvadurai & 1984 & $k_{s}=\frac{0.65 E_{s}}{B\left(1-\mu^{2}\right)}$ \\
\hline 7 & Bowles & 1988 & $k_{s}=40(F S) q_{a}$ \\
\hline 8 & Bowles & 1996 & $k_{s}=\frac{0.65 E_{s}}{B_{1}\left(1-\mu^{2}\right) m I_{f} I_{S}}$ \\
\hline 9 & Barounis et al. & 2013 & $k_{s}=100\left(q_{c}\right)$ \\
\hline
\end{tabular}

\section{Location overview and field tests}

All the data used in this paper is collected from an investigation project was carried on the southern Libyan desert in Gialo for constructing an oil station, this project demanded applying many field tests including fifteen Plate load tests, thirty CPTs, and thirty SPTs distributed equally over three different regions, namely: 6J, NC98 and Gialo iii, the fine sand formation of this regions is mainly the same but the densification of this sand varies between medium dense to dense. while the geologic formation [9] is Carbonate-marl-clay intercalations with gypsum interbeds near the top of Al Khums Fm. upper part of which may belong to Late Miocene; richly fossiliferous carbonates with subordinate sandy-marl intercalations of the upper part of Marada Fm., carbonate locally clastic-evaporitic sequence of Ar Rajmah Fm, carbonate-clay-marl, locally saliniferous and gypseous sequence of Al Jaghbub Fm; near-shore or continental beds in Sirte Basin.

\subsection{Plate load test and its limitations.}

One of the most popular in situ test is the plate load test, it is used to calculate the ultimate bearing capacity of the shallow foundations on the desired depth. Generally, the test can be performed by applying a vertical load on a standard plate, then recording the corresponding vertical displacement with measuring device such as dial gauges, a group of dial 
gauges with accuracy not less than $0.25 \mathrm{~mm}$ should be used, and then the average of these gauges readings recorded as the settlement due to this load, increasing the amount of load and recording the corresponding settlement many times until one of the following three conditions is reached, first the total settlement reaches ten percent of the plate diameter, second a peak load is reached, third the ratio of the settlement increment to the corresponding load increment reaches steady magnitude. The main disadvantages of the plate load test were carried out by Barounis et al., $[10,11]$ and it can be summarized as the following: a) the size of the plate is limited compared to the foundation size, so the pressure bulb of the plate is much smaller than that of the foundation, b) Time effect, since the test take short time, the results are sometimes not that reliable, c) water table effect, especially if the water table is above the footing level, so this water has to be pumped before applying the test, and this can increase the cost of the test, and d) the test needs well-trained technicians.

For this reason, an alternative in situ test might be used to Estimate the value of the subgrade coefficient, one such test is the Cone Penetration Test.

\subsection{Cone penetration test (CPT)}

Cone penetration test is frequently used for soil investigation projects, the main concept of this test is pushing a standard $35.7 \mathrm{~mm}$ diameter penetrometer through the soil, this penetrometer has a conical tip and an apex angle of $60^{\circ}$ to measure the soil resistance for penetration, the vertical force applied by the penetrometer stresses the soil to failure, this force divided by the base area gives the tip resistance qc in MPa, also the penetrometer has a sleeve friction located directly above the conical tip, and this friction sleeve measures the friction exerted by the soil fs, which is called sleeve friction, these are the main two parameters recorded from this test for each centimeter penetrated through the test, these two parameters are having very useful correlations that can be used to estimate almost every other parameter used in geotechnical engineering, the advantages of this test and what makes it favorable over the plate load test are discussed by Barounis et al., [12] Summarizing the main advantages as the following: a) Consuming less time, and low coast b) Excavation and dewatering works are not needed to perform this kind of test c) Can penetrate the soil to great depths, and the coefficient of subgrade reaction can be determined to every depth d) Records data for every centimeter and many different parameters can be calculated for each centimeter of the soil e) Reaches very high stress compared to plate load test

But the main limitations of this test are, it does not penetrate through hard soils and the refusal might occur in insufficient depth, and penetrating through the soil leads to soil failure, thus all the measured properties are in the failure condition.

\section{Aim of research}

The main aim of this paper is to estimate an empirical equation connecting the module of subgrade reaction with the tip resistance $\mathrm{q}_{\mathrm{c}}$ gathered from the CPT for non-cohesive sandy soil. And then to check the adequacy of this equation by comparing its results with the results obtained using the other well-known related equations.

\section{Critical review}

There aren't man formulas connecting the subgrade reaction with the cone resistance, however in 2013 Barounis et al. [12] introduced a methodology for estimating the subgrade coefficient from the CPT, they suggested that the penetration rate is conceptually equal to the vertical deformation, applying this concept to Winkler's module gives that $k_{s}=100 q_{c}$ for tests with penetration rate equals to $1 \mathrm{~cm} / \mathrm{s}$, although the vertical penetration might be used as an indication for vertical deformation, but they are not equal, imagine for the same soil two CPTs are performed with two different penetration rates, two different Ks coefficient will be obtained for the same soil and that is not reasonable. although an extensive comparison was made between Ks gathered from SPT and Ks gathered from CPT by Barounis and Menefy [13] this comparison should have included an experimental test that is more related to Ks such as CBR, consolidation, or in situ test such as plate load test to give a reference value instead of using SPT counts. The main disadvantages of using SPT for such as comparison are shown in the studies carried out lately by Alpaslan, N. [14]. As he examined the results of Ks obtained from three different approaches which are SPT blow counts, Bowles [7]. Equations, and Geophysical S Wave Velocity. The results showed that the correlation using the SPT counts to evaluate the subgrade reaction coefficient might yield misleading results especially when standard equipment and qualified persons are not used. On the other hand, the parameters affecting the subgrade reaction are studied earlier by Moayed and Janbaz [15] their studies showed that one such parameter is the size effect, there studies were carried on different clayey soils and they showed an inverse relationship between the subgrade reaction and the size of the foundation, this conclusion is supported by the results of this paper when it comes to the sandy soils, but both of these results are 
countered by the experimental studies carried out by Wael N. Abd Elsamee [16] as he stated that the subgrade reaction is increased as the size of foundation increased.

\section{Methodology for estimating the equation}

In order to estimate a correlation between the subgrade coefficient and the CPT readings, a set of data collected in the soil investigation project has been statistically analyzed using specialized software (Minitab).

Step-by-step detailed procedure for making the CPT and the plate load test are found in ASTM D 5778-12 [17] and ASTM D 1194-94 [18], respectively.

The subgrade coefficient was calculated from a $45 \mathrm{~cm}$ plate load test using a computer software named (Novo Lab), the plate test was conducted on the ground surface.

The tip resistance qc readings collected from the CPT is analyzed using computer software (CPTU-ACQ), and then the values of qc are plotted against the different values of Ks to estimate the best equation can be used to connect the variables, then to check the adequacy of the formula a series of comparisons is made with analytical software's which are (Microsoft Excel) and (Minitab). This comparison includes the results obtained from the empirical formula and the results of equations number $1,3,5,6,7$ in Table 1.

\section{Results and discussion}

The results of fifteen plate load tests analyzed with computer program (Novo Lab) are plotted on Y-axis against the result of tip resistance $\mathrm{q}_{\mathrm{c}}$ gathered from adjacent fifteen CPTs then the best fit curve is plotted using Microsoft Excel. Figure (1) shows the graph and the best-fit equation.

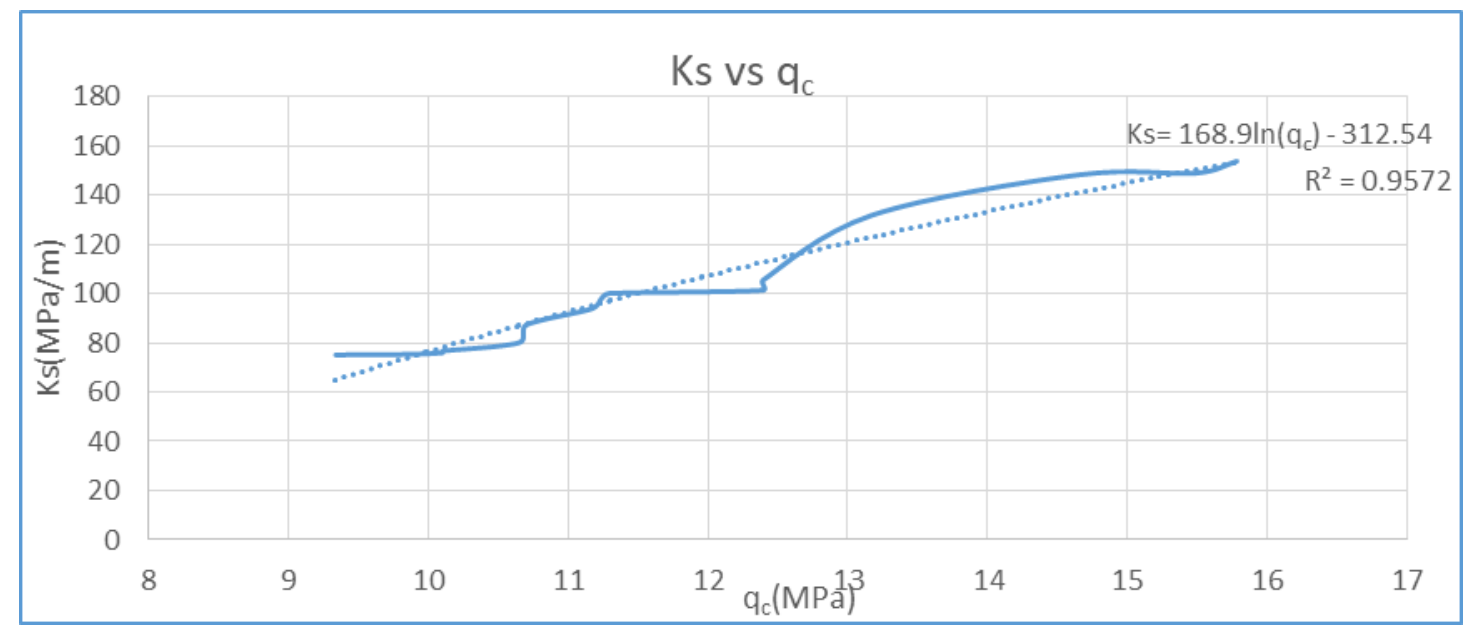

Figure 1 The values of subgrade reaction vs tip resistance from adjacent CPTs

Using this empirical equation to estimate the module of subgrade reaction from the tip resistance, then comparing the results obtained from this equation with the results calculated from the other well-known equations, figure (2) shows the different values of Ks estimated from each equation, it can be noted that the results obtained from this empirical equation are very close to the results obtained from equation 3 , the main reason for this close results is that the diameter of the plate used for the test is relatively close to the standard diameter suggested by Terzaghi which is $0.3 \mathrm{~m}$. Generally, the results obtained from the empirical equation lies in between the results obtained from the other equations. on the other hand, figure(3) shows the result obtained from the same equations, but for a foundation width equal to $1 \mathrm{~m}$ to study the effect of width on the equation and then the same comparisons are made, the results obtained from the empirical equation are varying widely from the results obtained from equation 3 and this shows the effect of width change on the empirical equation, also the values of Ks are relatively smaller than the values obtained before, and this concludes that the subgrade reaction decreases as the width of footing increases. On the other hand, the results obtained from equation 5 are matching the results of the empirical formula when the value of qc is relatively small, but for higher values of qc, difference between the two equations is noticed. The values of Ks estimated for $0.45 \mathrm{~m}$ width are relatively higher but they are still on the range specified by Braja M. Das [19] for dense soil as Table (3) shows. On the other hand, 
Minitab analysis are shown in figure (4) for analyzing the variance between the different equations, and to show the equations with nearly equal results graphically.

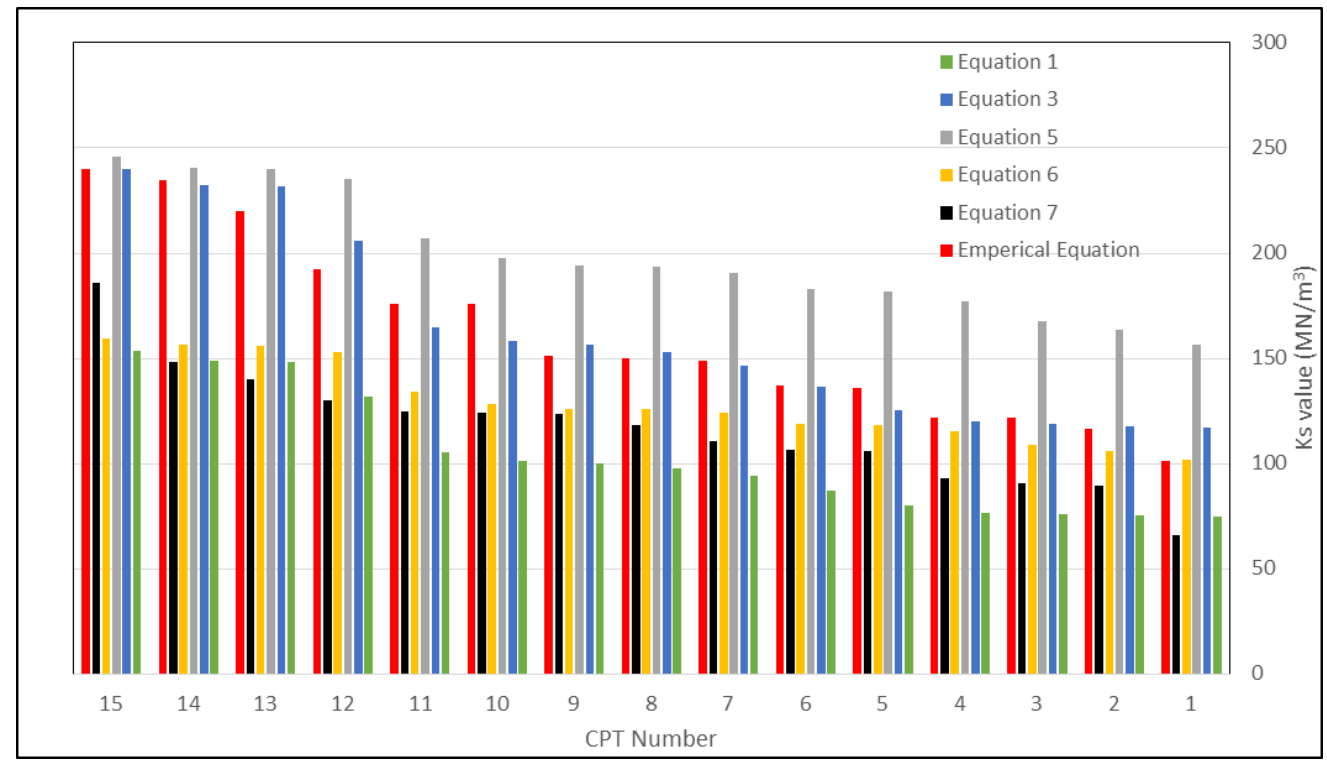

Figure 2 The values of subgrade reaction for each CPT for $b=0.45 \mathrm{~m}$

The final comparison is made between this empirical equation and equation number 9 which is considered to be the only equation depending on the same parameter qc, the method of comparison is to calculate the mean of all the other well-known equations and then to find the standard deviation from this mean, the results of this comparison are shown in Table 2.

Table 2 Comparison between the empirical equation \& equation number 9

\begin{tabular}{|l|c|c|}
\hline \multirow{2}{*}{} & \multicolumn{2}{|c|}{$\begin{array}{c}\text { Standard deviation } \\
\left(\mathbf{M N} / \mathbf{m}^{\mathbf{3}} \mathbf{)}\right.\end{array}$} \\
\cline { 2 - 3 } & $\mathbf{b}=\mathbf{0 . 4 5} \mathbf{~ m}$ & $\mathbf{b}=\mathbf{1} \mathbf{~ m}$ \\
\hline Empirical equation & \pm 22.3 & \pm 44.9 \\
\hline Equation number 9 & \pm 25.44 & \pm 51.1 \\
\hline
\end{tabular}




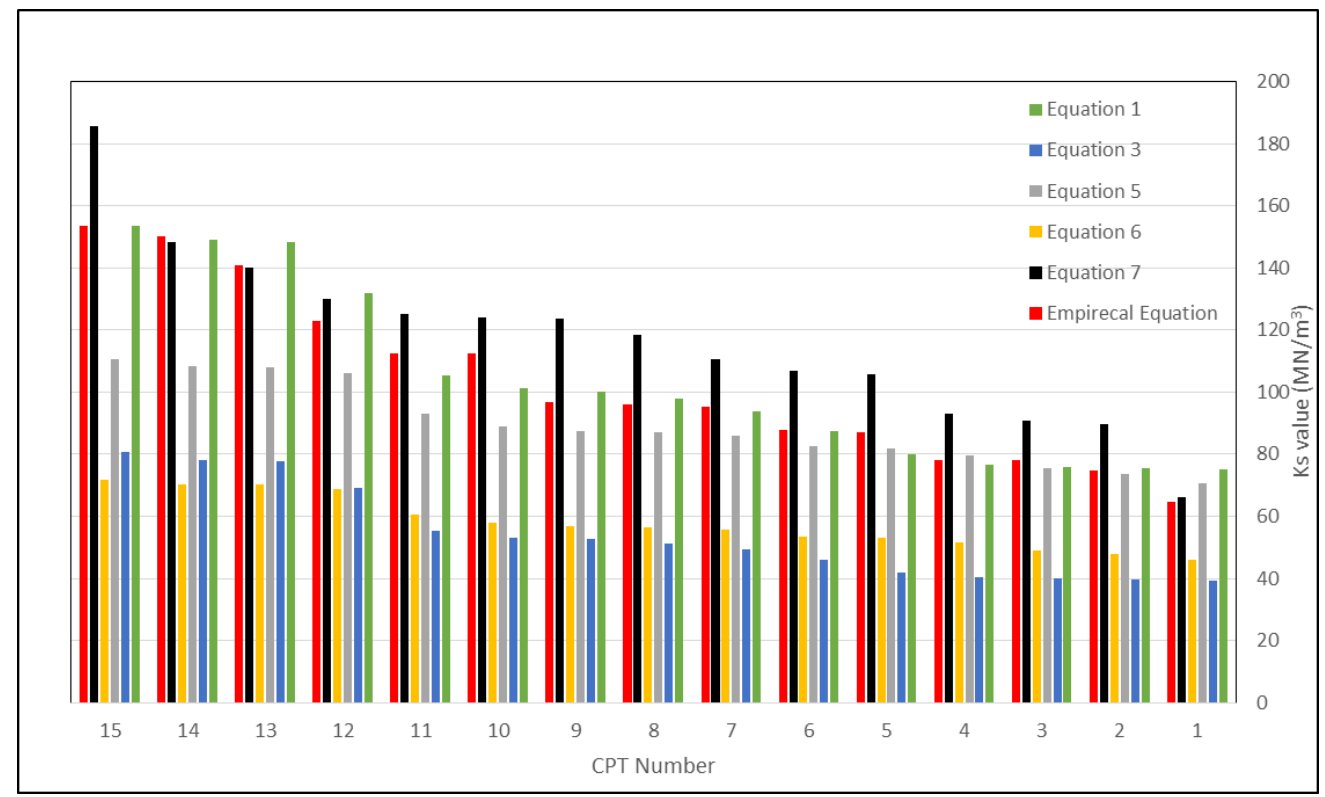

Figure 3 The values of subgrade reaction for each CPT for $b=0.45 \mathrm{~m}$

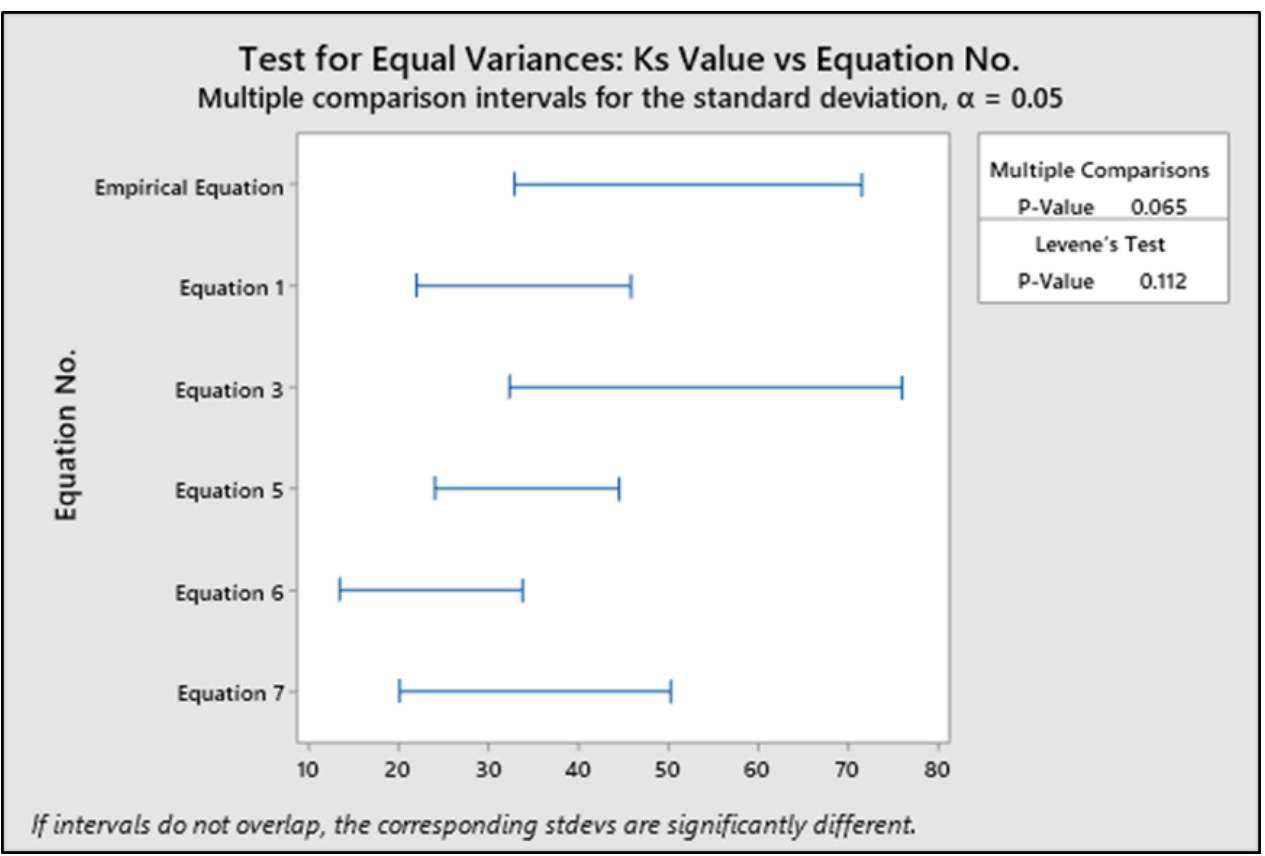

Figure 4 test for equal variances for $b=0.45 \mathrm{~m}$ 


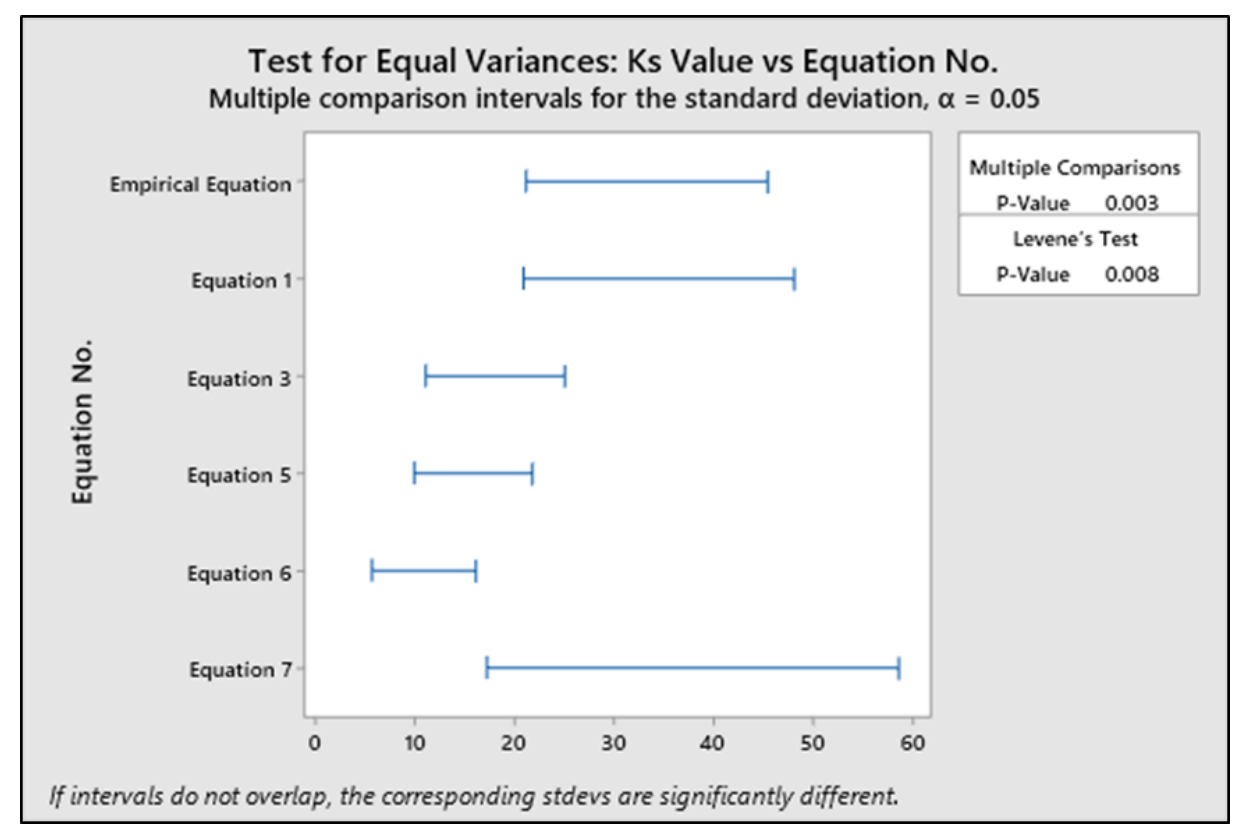

Figure 5 test for equal variances for $b=1 \mathrm{~m}$

Table 3 Typical Subgrade Reaction Values, $\mathrm{k}_{0.3}\left(\mathrm{k}_{1}\right)$

\begin{tabular}{|l|l|l|}
\hline Soil type & MN/m & lb/in ${ }^{3}$ \\
\hline Dry or moist sand: \\
\hline Loose & $8-25$ & $30-90$ \\
\hline Medium & $25-125$ & $90-450$ \\
\hline Dense & $125-375$ & $450-1350$ \\
\hline Saturated sand: \\
\hline Loose & $10-15$ & $35-55$ \\
\hline Medium & $35-40$ & $125-145$ \\
\hline Dense & $130-150$ & $475-550$ \\
\hline Clay: & $10-25$ & $40-90$ \\
\hline Stiff & $25-50$ & $90-185$ \\
\hline Very stiff & $>50$ & $>185$ \\
\hline Hard &
\end{tabular}

\section{Conclusion}

In order to derive a relation between the cone resistance $\mathrm{q}_{\mathrm{c}}$ and the module of subgrade reaction Ks, a data consist of fifteen plate load tests and thirty CPTs has been analyzed, the best-fit equation connecting these variables was found to be:

$\mathrm{Ks}=168.9 \ln \left(\mathrm{q}_{\mathrm{c}}\right)-312.54$

This equation is valid for medium to dense sand, and the results obtained from this equation are lying in between the boundaries specified by (Braja M. Das, 2016), finally to improve this equation the effect of the shape and depth on this equation should be studied since all the plate load tests are carried out by circular $45 \mathrm{Cm}$ diameter plate on the surface, on the other hand, similar equations for different types of soils might be conducted and the friction resistance obtained from the CPT might be included in such as equations. 


\section{Compliance with ethical standards}

\section{Acknowledgments}

The authors would like to thank Geo-Libya Engineering and Consultancy Company for providing the data used in this study. The contents of this paper do not necessarily reflect the official views or policies of Geo-Libya. The contents of this paper reflect the views of the authors who are responsible for the facts and the accuracy of the data presented herein. This research was technically supported by University of Benghazi.

\section{Disclosure of conflict of interest}

All authors would like to declare that there is no conflict of interest relevant to this article.

\section{References}

[1] Winkler E. Die lehre von elastizat and festigkeit (on elasticity and fixity). 1867; 182.

[2] Biot MA. Bending of infinite beams on an elastic foundation. Journal of Applied Physichs. 1937; (12): 155-164.

[3] Terzaghi K. Evaluation of coefficients of subgrade reaction. Géotechnique. 1955; 5(4): 297-326.

[4] Vesić AS. Beams on elastic subgrade and the Winkler's hypothesis. Proceedings of the 5th International Conference on Soil Mechanics and Foundation Engineering. 1961; 1: 845-850.

[5] Meyerhof GG, Baike LD. Strength of steel culverts sheets bearing against compacted sand backfill, Highway Research Board Proceedings. 1963; 30.

[6] Selvadurai APS. Elastic analysis of soil-foundation interaction. Developments in Geotechnical Engineering, Elsevier Scientific Publishing Co., Amsterdam. 1979; 17.

[7] Bowles JE. Foundation analysis and design 4th ed. McGraw Hill Book Company, New York. 1988.

[8] Bowles JE. Foundation analysis and design. 5th ed, United State of America: McGraw-Hill. 1996; 219-270 and 501-588.

[9] Industrial Research Center, Geological Research \& Mining Department. Geological Map of Libya. ER-Petro Consulting LTD., and Rotary International LTD. Hungary. 2009.

[10] Barounis N, Orr TLL, Barounis A, Neratzis P. Modulus of subgrade reaction for foundations on clay from consolidation tests. Proceedings of the European Conference of Soil Mechanics and Geotechnical Engineering, Madrid, Spain. 2007.

[11] Barounis N, Orr TLL, McMahon P, Barounis A. Modulus of subgrade reaction for foundations on clay from unconfined compression tests. Proceedings of the 17th International Conference on Soil Mechanics and Geotechnical Engineering, Alexandria, Egypt. 2009.

[12] Barounis N, Saul G, Lally D. Estimation of vertical subgrade reaction coefficient from CPT investigations: applications in Christchurch, Proceedings 19th NZGS Geotechnical Symposium. 2013.

[13] Barounis N, Menefy B. Estimation of vertical subgrade reaction modulus from CPT and comparison with SPT for a liquefiable site in Christchurch. 12th ANZ 2015 Conference on Geomechanics: Changing the Face of the Earth Geomechanics and Human. 22-25 February 2015.

[14] Alpaslan N. Comparison of Subgrade Reaction Coefficient Values Obtained With Different Approaches in Soil Investigations. European Journal of Science and Technology. 2021; (21): 223-229.

[15] Moayed RZ, Janbaz M. Subgrade reaction modulus of Tehran alluvium. Proceedings of the Institution of Civil Engineers, Geotechnical Engineering 164. 2011; 283-288.

[16] Wael N, Abd Elsamee. An Experimental Study on the Effect of Foundation Depth, Size and Shape on Subgrade Reaction of Cohessionless Soil. Scientific Research. 2013; 5: 785-795.

[17] ASTM D 5778-12 Standard Test Method for Electronic Friction Cone and Piezocone Penetration Testing of Soils. American Society for Testing Materials. 2021.

[18] ASTM D 1194-94. Standard Test Method for Bearing Capacity of Soil for Static Load and Spread Footings. American Society for Testing Materials. 2021. 
Global Journal of Engineering and Technology Advances, 2021, 09(02), 001-008

[19] Das BM. Principles of Foundation Engineering. 8th Ed. United State of America: Global Engineering: Timothy L. Anderson. 2016. 